\title{
Incorporación del Aprendizaje Basado en Proyectos en el Grado en Ingeniería de Obras Públicas: contexto, limitaciones y oportunidades
}

\author{
Ignacio Andrés-Doménech ${ }^{\mathrm{a} 1}$, Amalia Sanz-Benlloch ${ }^{\mathrm{a} 2}$, Eugenio Pellicer ${ }^{\mathrm{a} 3}$, M. Esther \\ Gómez-Martín $^{\mathrm{a} 4}$, Tomás Ruiz ${ }^{\mathrm{a} 5}$ \\ ${ }^{a}$ Universitat Politècnica de València, Escuela Técnica Superior de Ingeniería de Caminos, Canales y \\ Puertos, València, España. 11igando@hma.upv.es, 22asanz@upv.es, 33pellicer@upv.es, \\ ${ }^{4}$ mgomar00@upv.es, ${ }^{5}$ truiz@tra.upv.es.
}

\section{Resumen}

La Universitat Politècnica de València está apostando a nivel institucional por la incorporación del Aprendizaje Basado en Proyectos (ABP) en sus titulaciones. La Escuela Técnica Superior de Ingeniería de Caminos, Canales y Puertos está desarrollando un proyecto de innovación y mejora educativa para estudiar la implementación de esta metodología en el Grado en Ingeniería de Obras Públicas. En ese contexto, esta comunicación presenta los resultados de la primera fase del proyecto, el análisis del contexto externo e interno en el que se enmarca la iniciativa. En primer lugar, se analiza la incorporación del ABP en las titulaciones afines de otras escuelas de España, evidenciándose un panorama muy dispar en el ámbito nacional. Posteriormente, se analiza el contexto interno desde tres puntos de vista: la titulación, los estudiantes y los profesores. El Grado en Ingeniería de Obras Públicas es una titulación habilitante al ejercicio de la profesión de Ingeniero Técnico de Obras Públicas en España y, por tanto, sujeta a unos requisitos de verificación. Así, se presentan las restricciones derivadas de este requisito, se analizan los grados de libertad en el diseño actual de la titulación, identificando las oportunidades para la incorporación del ABP en los itinerarios de la titulación. Por otra parte, el análisis del contexto del alumnado se centra en estudiar la situación académica de los estudiantes potenciales que pueden participar en el proyecto piloto para garantizar la viabilidad de los mismos. Finalmente, se presenta el análisis del contexto respecto de los profesores, actores clave en el diseño de la actividad. Esta fase del proyecto concluye con la propuesta del piloto que se está diseñando para su implementación en el curso académico 2020/21. 
Incorporación del Aprendizaje Basado en Proyectos en el Grado en Ingeniería de Obras Públicas: contexto, limitaciones y oportunidades

Palabras clave: Aprendizaje basado en proyectos, ingeniería civil, obras públicas, trabajo en equipo, trabajo interdisciplinar.

\section{Introducción}

La Universitat Politècnica de València (UPV) puso en marcha en 2018 un plan conjunto de innovación educativa, el plan "Aprendizaje y Docencia” (A+D), que agrupa las diferentes acciones de innovación educativa en la universidad. Una de las líneas estratégicas de la UPV es la promoción del desarrollo de proyectos dirigidos de forma clara y directa a la mejora del aprendizaje (VRED-VECA, 2019). En 2019 se apuesta por un mayor liderazgo institucional en los procesos de innovación educativa, en particular, impulsando el Aprendizaje Basado en Proyectos (ABP) en las titulaciones de la UPV.

El ABP es una metodología de aprendizaje en la que los estudiantes aprenden principalmente haciendo, a través de la investigación y la exploración. El ABP representa un conjunto de tareas de aprendizaje basado en la resolución de problemas reales, que implica al alumno en el diseño y la planificación del aprendizaje y en la toma de decisiones que culminan en la realización de un producto final (Jones et al., 1997). Un reciente estudio del University College London destaca la necesidad de enfatizar las metodologías docentes centradas en el estudiante, potenciar la interdisciplinaridad y conectar el aprendizaje con la realidad profesional, cuestiones, todas ellas, en el centro del ABP (UCL, 2019).

La ingeniería civil es, por su propia realidad profesional, una disciplina en la que esta metodología docente encaja muy bien en el proceso enseñanza-aprendizaje. En efecto, "el proyecto" es uno de los productos por excelencia que el ingeniero civil realizará en el transcurso de su vida profesional. En este contexto, la Escuela Técnica Superior de Ingeniería de Caminos, Canales y Puertos (ETSICCP) de la UPV está liderando el proyecto de innovación y mejora educativa "Incorporación del Aprendizaje Basado en Proyectos en el Grado en Ingeniería de Obras Públicas”. El proyecto ha comenzado en el curso 2019/20 con el análisis del contexto, limitaciones y oportunidades y concluirá con la implantación, en el curso 2020/21, de la prueba piloto en el cuatrimestre A de $4^{\circ}$ curso, que se está diseñando en esta primera fase. 


\section{Contexto externo: el ABP en las escuelas de Ingeniería de Caminos}

La Conferencia de Directores de Ingeniería de Caminos, Canales y Puertos y de Ingeniería Civil (CODICAM) es una institución constituida por los directores de los 13 centros universitarios públicos españoles que imparten tanto el título universitario ofícial de Máster en Ingeniería de Caminos, Canales y Puertos que otorga las atribuciones profesionales de Ingeniero de Caminos, Canales y Puertos, como el título universitario oficial de Graduado en Ingeniería Civil (o denominación similar) que otorga las atribuciones de Ingeniero Técnico de Obras Públicas y que permite el acceso a dicho máster.

En el contexto de los centros educativos integrados en CODICAM, se elaboró y distribuyó a las Direcciones de las 13 Escuelas una encuesta (febrero de 2020) para conocer el nivel de implantación del ABP tanto en el título de Grado como en el de Máster (Fig. 1).

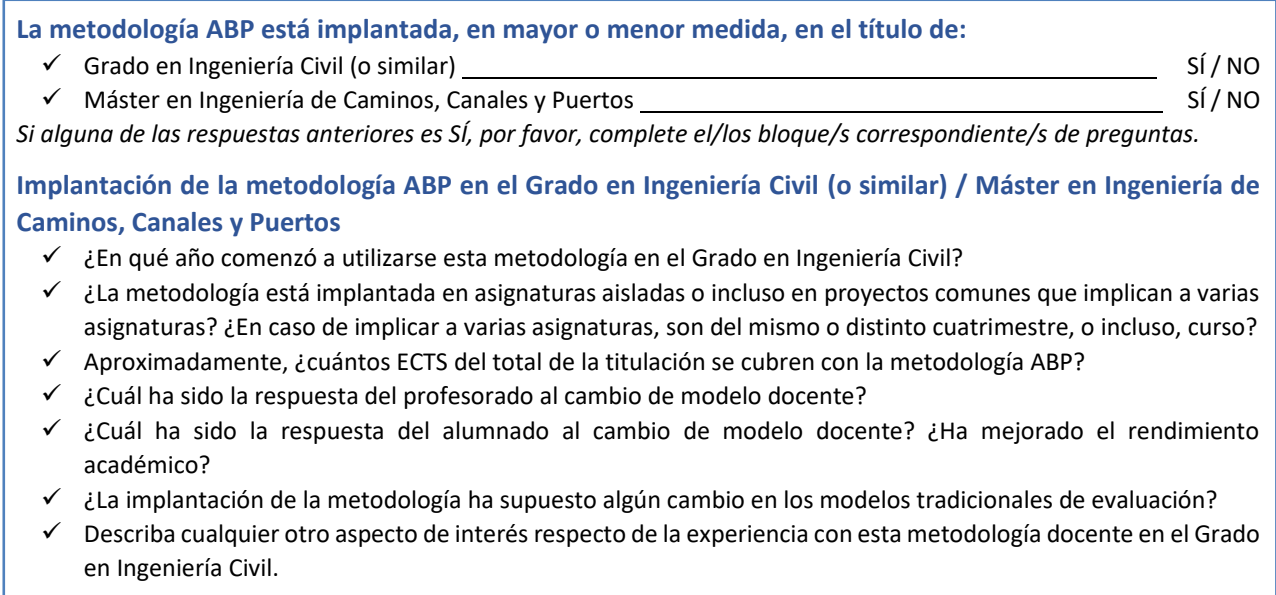

Fig. 1 Encuesta para el análisis del nivel de implantación del ABP en las Escuelas de Caminos y Politécnicas de España.

Los resultados más significativos de la encuesta, en lo relativo a los títulos de Grado en Ingeniería Civil (o similar), contexto de interés en el presente artículo, se sintetizan en la Tabla 1. Apenas la mitad de Escuelas tienen información disponible acerca de la implantación de esta metodología, lo cual ya deja entrever un grado de implantación muy discreto a nivel nacional.

Entre las escuelas que utilizan la metodología ABP, destaca la ETSICCP de Ciudad Real, pionera, con diferencia en esta metodología. La respuesta del alumnado en esta Escuela es totalmente satisfactoria, pese a la demanda de tiempo y estudio. En cuanto a la evaluación, 
Incorporación del Aprendizaje Basado en Proyectos en el Grado en Ingeniería de Obras Públicas: contexto, limitaciones y oportunidades

los trabajos proyectuales que siguen la metodología $\mathrm{ABP}$ se realizan en equipo, primando la evaluación contínua. En los casos en los que la trayectoria permite hacer comparaciones (caso de algunas asignaturas en la Universidad de Sevilla), se observan mejoras en el rendimiento académico al producirse el cambio de metodología.

Todos los casos que manifiestan utilizar la metodología destacan que los resultados se traducen, en una alta motivación de los estudiantes, el desarrollo de competencias transversales ligadas a las actividades, el trabajo en un entorno multidisciplinar y una alta valoración de la metodología docente por parte de los empleadores.

Tabla 1. Nivel de implantación del ABP en los Grados de Ingeniería Civil (o similar) en las Escuelas de Caminos y Politécnicas de España.

\begin{tabular}{|c|c|c|c|c|c|}
\hline Universidad & Escuela & $\begin{array}{l}\text { Usa ABP } \\
\text { en Grado }\end{array}$ & $\begin{array}{l}\text { Año } \\
\text { inicio } \\
\text { ABP }\end{array}$ & $\begin{array}{c}\text { ABP en asignaturas } \\
\text { aisladas o proyectos } \\
\text { comunes }\end{array}$ & $\begin{array}{c}\text { ECTS } \\
\text { cubiertos } \\
\text { con ABP }\end{array}$ \\
\hline Alicante & Escuela Politécnica Superior & Sí & $2012 / 13$ & Asig. aisladas & $18 / 240$ \\
\hline Burgos & Escuela Politécnica Superior & \multicolumn{4}{|c|}{ Sin información } \\
\hline Cadiz & Escuela Politécnica Superior de Algeciras & \multicolumn{4}{|c|}{ Sin información } \\
\hline Cantabria & ETSI Caminos, Canales y Puertos & \multicolumn{4}{|c|}{ Sin información } \\
\hline Castilla-la-Mancha & ETSI Caminos, Canales y Puertos & Sí & $2010 / 11$ & Proyecto común & $54 / 240$ \\
\hline Coruña & ETSI Caminos, Canales y Puertos & \multicolumn{4}{|c|}{ Sin información } \\
\hline Granada & ETSI Caminos, Canales y Puertos & \multicolumn{4}{|c|}{ Sin información } \\
\hline Oviedo & Escuela Politécnica de Mieres & Sí & $2018 / 19$ & Proyecto común & - \\
\hline Politécnica de Cartagena & ETSI Caminos, Canales y Puertos y Minas & No & - & - & - \\
\hline Politècnica de Catalunya & ETSE Camins, Canals i Ports & \multicolumn{4}{|c|}{ Sin información } \\
\hline Politécnica de Madrid & ETSI Caminos, Canales y Puertos & No & - & - & - \\
\hline Politècnica de València & ETSI Caminos, Canales y Puertos & Sí & - & Asig. aisladas & $24 / 240$ \\
\hline Sevilla & ETS de Ingeniería & Sí & $2013 / 14$ & Asig. aisladas & $36 / 240$ \\
\hline
\end{tabular}

En la ETSICCP de la UPV, existen, en el Grado en Ingeniería de Obras Públicas, asignaturas que de manera individual utilizan la metodología, mayoritariamente en $3^{\circ}$ y $4^{\circ}$.

Con la excepción de la ETSICCP de Ciudad Real, que diseñó su plan de estudios de Grado teniendo en cuenta explícitamente esta metodología, el resto de Escuelas que la utilizan lo hacen todavía de forma aislada en asignaturas $\mathrm{y}$, desde luego, no primando esta metodología en el título. Se están desarrollando distintas iniciativas que, aunque limitadas, ponen de manifiesto los buenos resultados que el ABP puede suponer en el aprendizaje del estudiante.

Puede por tanto concluirse que, aunque tímidamente, el ABP se va haciendo hueco en los títulos de Grado de las Escuelas. La experiencia que se está desarrollando en el Grado en Ingeniería de Obras Públicas de la UPV sigue esta senda, con el objetivo de consolidar el modelo. 


\section{Contexto interno: la ETSICCP de la UPV}

\subsection{La titulación}

El Grado en Ingeniería de Obras Públicas es una titulación habilitante al ejercicio de la profesión de Ingeniero Técnico de Obras Públicas y, por tanto, sujeta a unos requisitos de verificación recogidos en la Orden CIN/307/2009 (BOE, 2009). La orden establece los requisitos mínimos del plan de estudios, así como las competencias que el estudiante debe adquirir. Del total de 240 ECTS de la titulación, la orden prefija la distribución de 180 ECTS en los módulos de formación básica (60 ECTS), común a la rama civil (60 ECTS), de tecnología específica (48 ECTS) y TFG (12 ECTS). Los 60 ECTS restantes constituyen pues una holgura en el diseño de la titulación. En el caso del Grado en Ingeniería de Obras Públicas de la UPV, la configuración final por módulos refuerza significativamente el tamaño del módulo común a la rama (96 ECTS) y el de tecnología específica (54 ECTS).

El estudiante debe elegir el módulo de tecnología específica (construcciones civiles, hidrología o transportes y servicios urbanos) en el cuatrimestre B de tercer curso. De este modo, durante un curso y medio, el estudiante se forma en su itinerario específico. Esto resulta idóneo para plantear, en los intinerarios de especialidad, diseños curriculares coordinados que permitan la integración del ABP bien a escala de cuatrimestre, bien a lo largo de los 3 cuatrimestres de especialidad. Esta oportunidad es la que se ha querido aprovechar en el diseño de la prueba piloto que se está configurando para el curso 2020/21.

\subsection{Los estudiantes}

El número de estudiantes de nuevo ingreso en la titulación de Grado en Ingeniería de Obras Públicas es reducido. En el curso 2019/20, el número de estudiantes en cada itinerario de tecnología específica en el cuatrimestre $\mathrm{B}$ de $3^{\circ}$ curso de la titulación eran 21 en el itinerario de Construcciones Civiles, 18 en el de Transportes y Servicios Urbanos y 13 en el de Hidrología; de los cuales, sólo 4, 1 y 1, respectivamente, estaban cursando simultáneamente todas las asignaturas del cuatrimestre. Ante esta situación, se llegó a la conclusión de que no convenía diseñar el piloto para la implantación del $\mathrm{ABP}$ en el cuatrimestre $\mathrm{B}$ de $3^{\circ}$ curso, sino en el cuatrimestre A de $4^{\circ}$ curso. En este cuatrimestre, los estudiantes, aunque divididos en los tres itinerarios de especialidad, cursan una asignatura obligatoria común, “Taller de práctica profesional”, lo cual, como se verá más adelante, supone una muy buena oportunidad para el diseño de la prueba piloto. Además, esto resuelve la poca masa de estudiantes por itinerario, al quedar todos aunados por una asignatura común. Para el curso 2020/21, se espera una matrícula en esta asignatura de entre 30 y 40 estudiantes. 
Incorporación del Aprendizaje Basado en Proyectos en el Grado en Ingeniería de Obras Públicas: contexto, limitaciones y oportunidades

\subsection{Los profesores}

En diciembre de 2019 se presentó, por parte de la Escuela como coordinadora del proyecto, la idea de prueba piloto para el curso 2020/21 a los profesores implicados. Se organizaron reuniones por itinerarios de especialidad y conjuntas, para analizar la viabilidad del proyecto. Desde el primer momento, la acogida fue muy positiva por parte de los 10 profesores responsables implicados en la prueba piloto. Estas reuniones supusieron un impulso decisivo en el desarrollo del proyecto, permitiendo su materialización definitiva.

\section{Oportunidades y limitaciones para la implantación del proyecto}

El análisis del contexto presentado en el punto anterior ha permitido llevar a cabo un análisis de debilidades, amenazas, fortalezas y oportunidades (DAFO) del proyecto de incorporación del ABP en el Grado en Ingeniería de Obras Públicas (Fig. 2).

\begin{tabular}{|c|c|c|}
\hline & De origen interno & De origen externo \\
\hline 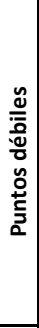 & $\begin{array}{l}\text { DEBILIDADES } \\
\text { homogéneamente de asignaturas del mismo curso. } \\
\text { hostudiantes no están matriculados ni exclusivamente ni } \\
\text { significativamente el absentismo estudiantil. } \\
\text { - El grado de implicación del profesorado es muy dispar. } \\
\text { La programación actual de actividades docentes (calendario y } \\
\text { horario) tiene muy pocos grados de libertad. }\end{array}$ & $\begin{array}{l}\text { AMENAZAS } \\
\text { representar una amenaza para la viabilidad de los proyectos. } \\
\text { Los estudiantes pueden llegar a percibir las propuestas como un } \\
\text { aumento en su carga lectiva. } \\
\text { Los profesores pueden cometer el error de introducir estas } \\
\text { innovaciones sin renunciar a métodos docentes tradicionales (por } \\
\text { ejemplo, exámenes). } \\
\text { dedicación a la iniciativa supone un incremento significativo de } \\
\text { esfuerzo sin reconocimiento docente. } \\
\text { Existe cierto inmovilismo por parte de un sector del profesorado. }\end{array}$ \\
\hline 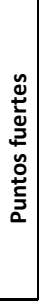 & $\begin{array}{l}\text { FORTALEZAS } \\
\text { - El profesorado implicado lo ha hecho de forma muy activa. } \\
\text { - El contexto de la Ingeniería Civil es muy adecuado para el } \\
\text { desarrollo de las propuestas de los PIME. } \\
\text { - La Dirección del Centro apuesta de forma decidida por impulsar } \\
\text { ambas iniciativas. }\end{array}$ & $\begin{array}{l}\text { OPORTUNIDADES } \\
\text { - El profesorado implicado reconoce la necesidad de plantear la } \\
\text { evolución de los planes de estudio vigentes a nuevos contextos. } \\
\text { - Los proyectos ponen en valor el trabajo multidisciplinar y en } \\
\text { equipo. } \\
\text { - El desarrollo de estas iniciativas promueve la coordinación } \\
\text { docente entre asignaturas. } \\
\text { - El desarrollo de estas iniciativas supondrá la mejora y } \\
\text { actualización de las Guías Docentes de las asignaturas. } \\
\text { - Los proyectos pueden aumentar la motivación y el rendimiento de } \\
\text { los estudiantes. }\end{array}$ \\
\hline
\end{tabular}

Fig. 2 Análisis DAFO para la implantación del proyecto "Incorporación del Aprendizaje Basado en Proyectos en el Grado en Ingeniería de Obras Públicas” (enero 2020).

Como ya ocurre en otras Escuelas, las oportunidades para los estudiantes son claras: aumento de la motivación, trabajo en equipo, integración de disciplinas, etc.; no obstante, existe la amenaza de no dimensionar correctamente las actividades propuestas y generar un sobreesfuerzo desmesurado. Por otra parte, deberá trabajarse la motivación inicial para evitar el desánimo y, en consecuencia, el absentismo que supondría el fracaso del proyecto. 
Desde el punto de vista del profesorado, la motivación detectada desde el primer momento compensa con creces las posibles amenazas o debilidades que, por el momento, no han aparecido. El profesor va a tener que aprender a coordinarse con sus compañeros de curso, cuestión que, con el modelo tradicional, ocurre sólo anecdóticamente.

Desde el punto de vista institucional, el apoyo e impulso del proyecto por parte de la Escuela aparece como un elemento decisivo para su desarrollo con ciertas garantías de éxito.

\section{Idea conceptual para la prueba piloto}

El proyecto piloto se ha planteado en el cuatrimestre A de $4^{\circ}$ curso. La propuesta de proyecto coordinado se plantea con el nexo común a todos los estudiantes en la asignatura "Taller de práctica profesional". En esta asignatura, el estudiante aprende los contratos de servicios y obras, los documentos del proyecto y su alcance, así como la valoración de las obras, todo ello en el contexto del ciclo de vida de la infraestructura. Así, esta asignatura resulta idónea para nutrirse de resultados tecnológicos concretos, desarrollados en las asignaturas tecnológicas correspondientes de cada itinerario (Fig. 3).

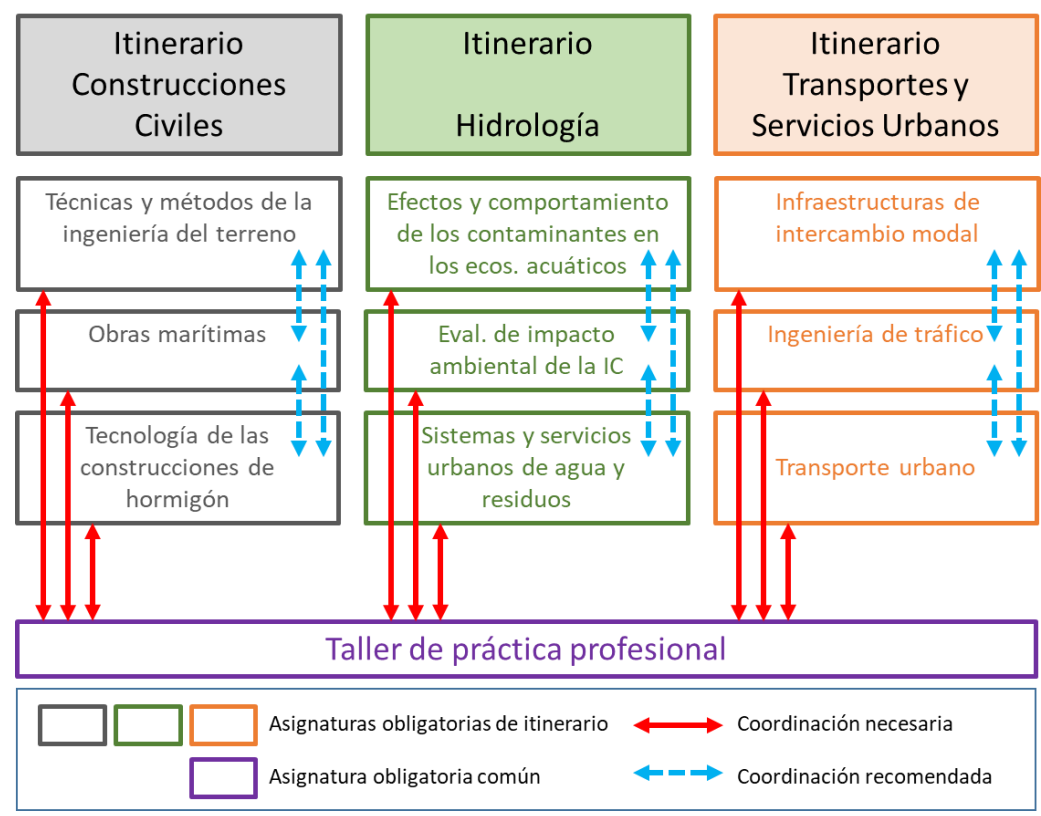

Fig. 3 Esquema conceptual de la prueba piloto para la implantación coordinada el ABP en el cuatrimestre A de cuarto curso del Grado en Ingeniería de Obras Públicas. 
Con este esquema de coordinación, se pretende adoptar un ejemplo concreto de proyecto de infraestructuras, a partir del cual, cada una de las 9 asignaturas tecnológicas obligatorias de especialidad pueda trabajar total o parcialmente sus aplicaciones prácticas para que, a continuación, éstas sean adoptadas por "Taller de práctica profesional” para el desarrollo conjunto del proyecto. Los estudiantes de cada itinerario trabajarán en sus asignaturas tecnológicas obligatorias de especialidad ciertos aspectos concretos del proyecto y los compartirán, con el resto de compañeros de las otras dos especialidades, en "Taller de práctica profesional". De este modo, el trabajo común en "Taller de práctica profesional" se asemejará a la práctica profesional real de una empresa de consultoría de ingeniería civil: el trabajo realizado en las asignaturas tecnológicas obligatorias de especialidad correspondería a la producción propia de los departamentos especializados de la empresa, mientras que el trabajo desarrollado en "Taller de práctica profesional" aúna todo este trabajo para su puesta en común en el proyecto completo.

Durante los meses de enero a julio de 2020, Escuela y profesores implicados, están trabajando en la definición y coordinación de actividades para el desarrollo del proyecto, con el objetivo de implantarlo en el curso 2020/21. En septiembre de 2020 se iniciará la docencia en $4^{\circ}$ curso del Grado con la metodología porpuesta, surante todo el primer cuatrimestre. En el segundo cuatrimestre, se analizará el grado de cumplimiento de los objetivos planteados y, en su caso, la posible implantación al resto de la titulación.

\section{Conclusiones}

La ETSICCP de la UPV ha apostado por la incorporación coordinada del ABP en su titulación de Grado en Ingeniería de Obras Públicas. Esta titulación resulta idónea para incorporar esta metodología en la parte final de la misma, donde los estudiantes siguen uno de los 3 itinerarios de especialidad. Con la implantación de la prueba piloto en el curso 2020/21, se espera comprobar los resultados satisfactorios que ya han documentado otras universidades españolas con la aplicación de esta metodología, resultando todo ello en la satisfacción, no sólo del alumnado, sino también del profesorado implicado.

\section{Agradecimientos}

Los autores agradecen la financiación recibida de la Universitat Politècnica de València a través del proyecto de innovación y mejora educativa PIME/19-20/149. 


\section{Referencias}

Jones, B. F., Rasmussen, C. M., \& Moffitt, M. C. (1997). Psychology in the classroom: A series on applied educational psychology.Real-life problem solving: A collaborative approach to interdisciplinary learning. Washington DC. American Psychological Association. https://doi.org/10.1037/10266-000

Orden CIN/307/2009, de 9 de febrero, por la que se establecen los requisitos para la verificación de los títulos universitarios oficiales que habiliten para el ejercicio de la profesión de Ingeniero Técnico de Obras Públicas, BOE núm.42 § 2736 (2009)

UCL Center for Engineering Education (2017). Innovations in Engineering Education. Inspiring \& Preparing Our Engineers for the 21st Century. UCL Center for Engineering Education \& Lloyd's Register Foundation. Recuperado 12/06/2020, de: https://www.ucl.ac.uk/centre-for-engineeringeducation/sites/centre-for-engineering-education/files/ucl_cee lrf_report_0.pdf

Vicerrectorado de Recursos Digitales y Documentación -Vicerrectorado de Estudios, Calidad y Acreditación (2019). Innovación educativa: convocatoria Aprendizaje + Docencia 2019. Recuperado 12/06/2020, de https://www.upv.es/contenidos/ICEP/info/Convocatoria 2019 C.pdf 